\title{
Role of drug transporters in drug development: a qualitative and quantitative approach
}

\author{
Sumit Basu, Abdul Naveed Shaik ${ }^{*}$ \\ Guest Editors; ADMET \& DMPK \\ Center for Pharmacometrics and Systems Pharmacology, University of Florida, Orlando, Florida. USA \\ *Corresponding Author: E-mail: naveedshaik@gmail.com; Tel.: +1-407-313-7009; Fax: +1-407-313-7030 \\ Received: June 21, 2017; Published: June 22, 2017
}

Through last two decades, transporters have been recognized as one of the important elements which plays an important role to regulate the ADME (Absorption, Distribution, Metabolism and Excretion) properties of drugs and xenobiotics. To evaluate the role of the transporters in drug development, an International Transporter Consortium (ITC) discussed about the identification of the clinically relevant transporters, application of these transporters in different drug-drug interaction (DDI) studies and the different criteria to determine whether additional DDI studies are required. Owing to the increase of transporter research studies in the last decade and the emphasis of the transporter mediated DDI by different regulatory agencies (FDA, EMA and PMDA), the incorporation of transporter related information in the different drug "Label" has increased from $24 \%$ to $56 \%$ within five years. In addition, the transporter related post marketing studies became quite important to address different questions regarding dose optimization and in the revision of drug product labelling.

In general, the role of transporters varies depending on their function and localization within the gut lumen, liver, kidney and brain. Depending on the directions of the transport, they can be classified as either efflux or influx/uptake. Usually efflux transporters shuttle compounds out of the cell against the concentration gradient whereas the uptake transporters direct compounds into the cells along the concentration gradient. Since many transporters have the ability to recognize more than one substrate, different types of DDI have been noticed due to the inhibition and induction of the function of the transporters. In addition, many transporters demonstrated genetic polymorphisms which may affect their function.

Traditionally, in vitro tools and preclinical animal experiments have often been used to assess the role of efflux transporters in drug development. Carefully designed and properly planned studies can give a clear understanding of the intrinsic functions and attributes of the study. The accumulation of the current in vitro and preclinical animal knowledge base is a conscious effort to link the clinical endpoints with the early developmental work which ultimately will help to identify the effect of drug transporters in the clinical settings. However, owing to the broad substrate specificity as well as significant overlap of inhibitor specificity, one should always investigate the experimental design and the results to determine the combined effects of particular transporters. Similarly, naturally occurring genetic variabilities arising from 
genetic polymorphisms can also affect both drug absorption and disposition which often results in inconclusive results. Although the lack of ideal probes and inhibitors has confounded the transporter data interpretations for intestinal absorption, the role of efflux in other organs (blood-brain-barrier) is better understood.

With the increasing interest in drug transporters from pharmaceutical companies and regulatory authorities alike and following the success of a previous special issue on drug metabolism [1] as well as a special issue on drug transporters and permeability $(2015$, volume 3 , issue 1$)$, ADMET \& DMPK devoted a special issue on "Role of drug transporters in drug development: a qualitative and quantitative approach" with the focus to explore the current trend in the uses of these transporters at various stages of drug discovery and development. We received a few original articles and two review articles, which covered various areas including, uric acid transporters and its inhibitors in gout, perillyl alcohol mediated abrogated membrane transport in Candida albicans, use of different fluorescent organic cations for screening against OCT2 transporters and expression of drug transporters in human skin and its uses in drug development. One particular submission of a great interest was "Consensus rank orderings of molecular fingerprints illustrate the 'most genuine' similarities between marketed drugs and small endogenous human metabolites, but highlight exogenous natural products as the most important 'natural' drug transporter substrates" where the authors S. O'Hagan and D.B. Kell brought about the similarities between small endogenous metabolites and marketed drugs.

These submissions clearly indicate the utilization of various drug transporters at different stages of drug discovery and development. As there has been significant interest in this special issue focussing on drug transporters we will follow with a second edition of the special issue to accommodate other submissions.

\section{References}

[1] A.N. Shaik, Changing trends in use of hepatocytes and microsomes for evaluating metabolism studies in drug discovery, ADMET and DMPK 4 (2016), 60-61. 